\title{
Robust Scale Estimation for the Generalized Gaussian Probability Density Function
}

\author{
Rozenn Dahyot ${ }^{1}$ and Simon Wilson ${ }^{2}$
}

\begin{abstract}
This article proposes a robust way to estimate the scale parameter of a generalised centered Gaussian mixture. The principle relies on the association of samples of this mixture to generate samples of a new variable that shows relevant distribution properties to estimate the unknown parameter. In fact, the distribution of this new variable shows a maximum that is linked to this scale parameter. Using nonparametric modelling of the distribution and the MeanShift procedure, the relevant peak is identified and an estimate is computed. The whole procedure is fully automatic and does not require any prior settings. It is applied to regression problems, and digital data processing.
\end{abstract}

\section{Introduction}

Many problems in computer vision involve the separation of a set of data into two classes, one of interest in the context of the application and the remaining one. For instance, edge detection in images requires the thresholding of the gradient magnitude to discard noisy flat areas from the edges. The challenge is then to automatically select the appropriate threshold (Rosin, 1997).

Regression problems also involve the simultaneous estimation of the variance or standard deviation of the residuals/errors. The presence of a large number of outliers makes difficult the estimation of the parameters of interest. Performance of robust estimators is highly dependent on the setting of a threshold or scale parameter, to separate the good data (inliers) that fit the model, from the gross errors (outliers) (Chen and Meer, 2003). The scale parameter, needed in M-estimation and linked to the scale parameter of the inliers residuals, is often set a priori or estimated by the Median Absolute Deviation. Applications of robust regression (Chen and Meer, 2003) are, for instance, line fitting (Wang and Suter, 2004), or camera motion estimation (Bouthémy et al., 1999). Those estimates also require the setting of a threshold (or scale parameter) to discard gross errors (outliers) from the relevant ones (inliers).

This paper proposes a solution for the estimation of the scale parameter that can be used iteratively along with location parameter estimation (Miller and Stewart, 1996; Chen and Meer, 2003; Wang and Suter, 2004) when there are many outliers, by assuming a

\footnotetext{
${ }^{1}$ School of Computer Science and Statistics, Trinity College Dublin, Ireland; dahyotr@tcd.ie

${ }^{2}$ School of Computer Science and Statistics, Trinity College Dublin, Ireland; swilson@tcd.ie
} 
mixture model. This method is based on the definition of two random variables $Y$ and $Z$ computed from the samples of variable $X$ using a non-linear transformation. The distributions of those new variables have properties that allow us to define new estimates for the scale parameter. The computation of those new estimates requires us to detect a particular maximum over the distribution of the new variables. This is achieved using nonparametric kernel-based modelling of probability density functions. The resulting method is then both robust and unsupervised.

Section 2 presents related works. Section 3 presents our new estimates. Section 4 presents the computation. Paragraph 5 proposes to use this procedure for scale estimation iteratively with a location parameter estimation. Section 6 presents experimental results showing the robustness of the approach, and an application to robust object recognition.

\section{Related works}

Several works have been carried out on robust regression in the vision community (Stewart, 1999), offering complementary views to statistics (Huber, 1981; Hampel et al., 1986). Wang has recently proposed a clear overview in both domains (Wang, 2004), underlining several differences. In particular, in the vision community, the breakdown point is usually expected far below $50 \%$ of outliers to deal with real world applications, and proofs of robustness are usually inferred through experimental results. In statistics, formal proofs of robust estimator properties usually prevail (even if those are only valid under strict assumptions that may never be encountered in practice) as it provides insights into the approaches (Hampel et al., 1986).

We consider the problem of robust regression:

$$
v=f(\mathbf{u}, \theta)+\epsilon
$$

The mapping function $f$ is assumed to be described by the vector $\theta$ (location parameter). From a set of observations $\left\{\left(v_{i}, \mathbf{u}_{i}\right)\right\}$, the goal of regression is to estimate $\theta$.

Maximum likelihood is a popular estimation method. It relies on the modelling of the probability density function of the residual $\epsilon$ that expresses the error between each observation and its prediction by the mapping. Standard parametric modellings for the pdf of the residuals include Gaussian and Laplacian density functions (Hasler et al., 2003). Those models fail when gross errors or outliers occur in the observations. In this case, the pdf of the residuals can be expressed as a mixture:

$$
\mathcal{P}(\epsilon \mid \theta, \sigma)=\mathcal{P}(\epsilon \mid \mathcal{C}, \theta, \sigma) \cdot \mathcal{P}(\mathcal{C})+\mathcal{P}(\epsilon \mid \overline{\mathcal{C}}) \cdot \mathcal{P}(\overline{\mathcal{C}})
$$

where $\mathcal{C}$ is the inlier class for the model designed by the location parameter $\theta$ and $\sigma$ the scale parameter. The distribution of the inliers $\mathcal{P}(\epsilon \mid \mathcal{C}, \theta, \sigma)$ is modelled by a parametric distribution, e.g. centered Laplacian (Hasler et al., 2003) or Gaussian (Wang and Suter, 2004), depending on the location parameter $\theta$ to be estimated and the scale parameter $\sigma$ also usually unknown. Those parametric models usually offers a good description in real world applications.

Two main strategies have been proposed to overcome the lack of robustness. The first weights the residuals in the objective function to lessen the influence of gross error on 
the estimate. Weights are then a function of the residuals. The second approach consists in using sampling strategies and in estimating from several randomly selected subsets of observations. The final selection is then performed by comparing each estimate and keeping the optimal one. In a way, it can be seen as a similar approach to weighting the residuals in taking null weights on left out data at each round. However, this approach is different as weights are set randomly on data and do not depend on the values of the residuals. M-estimators or R-estimators are examples of objective functions that use a weighting strategy (Huber, 1981; Hampel et al., 1986). By their efficiency and their rather low computation cost, M-estimators in particular, have been widely applied to many computer vision applications such as camera motion estimation (Odobez and Bouthémy, 1995), object class learning (la Torre and Black, 2001), object detection (Dahyot et al., 2004a) and recognition (Black and Jepson, 1998). Using sampling has been made popular by Fischler and Bolles with the RANSAC estimator (Fischler and Bolles, 1981). It has been successfully applied to camera calibration and image matching (Fischler and Bolles, 1981).

Both the M-estimator and RANSAC depend on the scale parameter $\sigma$ that needs to be robustly assessed along with $\theta$. Statistical solutions for the scale estimate include in particular the Median Absolute Deviation (Hampel et al., 1986) and Least Median Square (Miller and Stewart, 1996). Those methods have been used for online estimation from current observations. It can also be set by hand (Fischler and Bolles, 1981), or learned a priori off-line, and then set once and for all in the context of specific applications (Dahyot et al., 2000; Hasler et al., 2003). For the modelling of the distribution of outliers, $\mathcal{P}(\epsilon \mid \overline{\mathcal{C}})$, nonparametric representation using histograms have also been proposed (Hasler et al., 2003) to define a more dedicated M-estimator for image matching.

However, in most applications, the scale parameter cannot be inferred, and needs to be estimated in parallel to $\theta$ with a much lower breakdown than $50 \%$. Recently several strategies involving nonparametric representations of the distribution of the residuals $\mathcal{P}(\epsilon \mid \theta, \sigma)$ have been proven efficient. For instance, Chen et al. (Chen and Meer, 2003) proposed to re-express the M-estimator objective function on the residuals, as a kernel density like estimator where the scale parameter is substituted by a bandwidth. After localising the main central peak, surrounding basins of attraction are detected heuristically and are used as thresholds to separate inliers from outliers. The segmented inliers are then processed further to produce an estimate of $\theta$. Similarily in (Wang and Suter, 2004), the distribution of the residuals is modelled using a nonparametric kernel modelling. The central peak is assumed to be corresponding to the inliers, and is isolated from the rest of the pdf using a Meanshift based algorithm searching for surrounding valleys. From this rough classification, the scale parameter is then robustly estimated by computing the Median. This two step scale estimation (TSSE) is coupled with a location parameter $\theta$ estimation in a similar fashion to RANSAC (Fischler and Bolles, 1981).

However looking for minima in pdf where few data occur (Chen and Meer, 2003; Wang and Suter, 2004), is a more difficult operation than looking for maxima. Searching to maximize a pdf is also a more natural way for parameter estimation. We then propose another nonparametric approach in the next section that estimates the unknown scale parameter by searching for particular maxima in specific distributions. Another difference from (Chen and Meer, 2003; Wang and Suter, 2004) is that only a maximum localisation is necessary to infer the scale parameter. It is similar to the Hough transform approach 
in that peaks in the estimated distribution are directly related to the unknown parameters (Goldenshluger and Zeevi, 2004).

\section{New estimates for $\sigma$}

Another approach for scale parameter estimation has been proposed in (Dahyot et al., $2004 \mathrm{~b}$ ) based on some properties of independent samples from a Gaussian distribution. Indeed assuming a Gaussian distribution for the inliers, the distribution of square root of the sum of squares of several independent samples is a $\chi$ distribution that shows a maximum directly related to the scale parameter. This approach is further studied in this article.

\subsection{Assumptions}

Let us define the random variable $X$ that follows a two class mixture model. We call inliers the data that belong to the class of interest: $x \in \mathcal{C}$, and outliers the other data $x \in \overline{\mathcal{C}}$. It is possible to make some relatively loose assumptions about the distribution of the class $\mathcal{C}$ of interest, to allow its statistics to be estimated from an observed mixture distribution $\mathcal{P}_{X}(x)$ (Hasler et al., 2003). In this work, we assume the distribution of the inliers to be a Generalized centered Gaussian (Aiazzi et al., 1999):

$$
\begin{aligned}
& \mathcal{P}_{X}(x \mid \mathcal{C}, \sigma)=\frac{1}{2 \Gamma(\alpha) \cdot \alpha \cdot \beta^{\alpha}} \exp \left[\frac{-|x|^{1 / \alpha}}{\beta}\right] \\
& \text { with } \beta=\sigma^{1 / \alpha} \cdot\left[\frac{\Gamma(\alpha)}{\Gamma(3 \alpha)}\right]^{1 /(2 \alpha)}
\end{aligned}
$$

Setting $\alpha=1$ (Laplacian law) and $\alpha=1 / 2$ (Gaussian law) in equation (3.1), are two popular hypotheses (Hasler et al., 2003; Dahyot et al., 2004b). Assuming the shape parameter $\alpha$ known, we focus on the estimation of the scale $\sigma$.

\subsection{Definition of new variables}

The variables $Z=\sum_{n=1}^{\mathbf{n}}\left|X_{n}\right|^{1 / \alpha}$ and $Y=Z^{\alpha}$ are defined with independent random variables $X_{n}$ that follow the same probability density function (3.1).

For $\mathbf{n}=1$ and $Z=|X|^{1 / \alpha}$, the pdf $\mathcal{P}_{Z}(z \mid \mathcal{C})$ corresponds to the gamma distribution:

$$
\mathcal{G}_{Z \mid(\alpha, \beta)}(z)=\frac{z^{\alpha-1}}{\Gamma(\alpha) \cdot \beta^{\alpha}} \exp \left[-\frac{z}{\beta}\right], \quad z \geq 0
$$

When $\mathbf{n}>1$, the variable $Z$ is then defined by the sum of i.i.d. variables that follow a gamma distribution. Using the characteristic function of the gamma distribution $\Phi(t)=$ $(1-\imath \beta t)^{-\alpha}$, the characteristic function of $\mathcal{P}_{Z}(z \mid \mathcal{C}, \sigma)$ is:

$$
\Phi_{Z}(t)=\prod_{n=1}^{\mathbf{n}} \Phi(t)=(1-\imath \beta t)^{-\mathbf{n} \alpha}
$$


By Inverse Fourier transform, the p.d.f $\mathcal{P}_{Z}(z \mid \mathcal{C}, \sigma)$ is the gamma function $\mathcal{G}_{Z \mid(\mathbf{n} \alpha, \beta)}(z)$. From the distribution of $Z$, it is easy to infer the pdf of $Y$ :

$$
\mathcal{P}_{Y}(y \mid \mathcal{C}, \sigma)=\frac{y^{(\mathbf{n}-1)}}{\alpha \cdot \Gamma(\mathbf{n} \alpha) \cdot \beta^{\mathbf{n} \alpha}} \exp \left[\frac{-y^{1 / \alpha}}{\beta}\right], y \geq 0
$$

The maximum of the distributions $\mathcal{P}_{Z}(z \mid \mathcal{C}, \sigma)$ and $\mathcal{P}_{Y}(y \mid \mathcal{C}, \sigma)$ can be then computed (ignoring the solution $z=0$ as a special case):

$$
\left\{\begin{array}{l}
Z_{\max \mathcal{C}}=\beta \cdot(\mathbf{n} \alpha-1), \mathbf{n} \alpha>1 \\
Y_{\max \mathcal{C}}=[(\mathbf{n}-1) \alpha \beta]^{\alpha}, \mathbf{n}>1
\end{array}\right.
$$

Those maxima depend on the parameter $\sigma$ by definition of $\beta$ (cf. eq. (3.1)).

\subsection{New estimates for $\sigma$}

From equation (3.5), we propose to estimate $\sigma$ using:

$$
\left\{\begin{array}{cc}
\sigma_{Z}=\left(\frac{Z_{\max \mathcal{C}}}{\mathbf{n} \alpha-1}\right)^{\alpha}\left[\frac{\Gamma(3 \alpha)}{\Gamma(\alpha)}\right]^{1 / 2}, & \mathbf{n} \alpha>1 \\
\sigma_{Y}=\frac{Y_{\max \mathcal{C}}}{(\mathbf{n}-1)^{\alpha} \cdot \alpha^{\alpha}}\left[\frac{\Gamma(3 \alpha)}{\Gamma(\alpha)}\right]^{1 / 2}, & \mathbf{n}>1
\end{array}\right.
$$

The maximum of the distributions of $Y$ and $Z$ has first to be located. This can be made difficult by outliers occurring in the observations such that the observed distribution for $X$ is a mixture (as for $Y$ and $Z$ ):

$$
\mathcal{P}_{X}(x \mid \sigma)=\mathcal{P}_{X}(x \mid \sigma, \mathcal{C}) \cdot \mathcal{P}_{X}(\mathcal{C})+\mathcal{P}_{X}(x \mid \overline{\mathcal{C}}) \cdot \mathcal{P}_{X}(\overline{\mathcal{C}})
$$

Depending on the proportion and the values of the outliers, the localisation of the maximum needed in the estimation gets more difficult. We assume that the relevant maximum for the estimation is the closest peak to zero in the distributions $\mathcal{P}_{Y}(y \mid \sigma)$ and $\mathcal{P}_{Z}(z \mid \sigma)$. Note that robust estimation using M-estimator for Gamma distribution has been proposed in the literature (Marazzi A., 1996). But this nonparametric method is shown to be more robust in Section 6.

\subsection{Remarks}

Figure 1 presents the pdfs of the inlier class for $Y$ and $Z$. Instinctively, the higher the maximum, the better its localisation should be in the mixture of observations. A priori, considering the density of variable $Y$ for the estimation of the scale parameter should then perform better than the variable $Z$. However on the other hand, the transformation from $X$ to $Z$ is spreading the range of variations of the observations, and consequently decreasing locally the density of the outliers.

Expressions of the maxima are given by:

$$
\left\{\begin{array}{l}
\mathcal{P}_{Z}\left(Z_{\max } \mathcal{C} \mid \mathcal{C}, \sigma\right)=\frac{(\mathbf{n} \alpha-1)^{\mathbf{n} \alpha-1}}{\Gamma(\mathbf{n} \alpha) \cdot \beta} \exp [-(\mathbf{n} \alpha-1)] \\
\mathcal{P}_{Y}\left(Y_{\max } \mathcal{C} \mid \mathcal{C}, \sigma\right)=\frac{((\mathbf{n}-1) \alpha)^{(\mathbf{n}-1) \alpha}}{\alpha \cdot \Gamma(\mathbf{n} \alpha) \beta^{\alpha}} \exp [-(\mathbf{n}-1) \alpha]
\end{array}\right.
$$






Figure 1: Probability density functions $\mathcal{P}_{Y}(y \mid \sigma, \mathcal{C})$ and $\mathcal{P}_{Z}(z \mid \sigma, \mathcal{C})(\mathbf{n}=3$ and $\alpha=0.5)$.

\section{Robust non-parametric estimation}

Section 4.1 gives details on the computation of samples of the variable $Y$ and $Z$ from the samples of $X$. Paragraph 4.2 presents the nonparametric approach taken to perform the computation of the relevant maxima in the estimation of the scale parameter.

\subsection{Computing samples}

From a set of observed independent samples $\mathcal{B}_{x}=\left\{x_{i}\right\}_{i \in\{1 \cdots \mathrm{i}\}}$, we need first to compute the samples $\mathcal{B}_{z}=\left\{z_{j}\right\}_{j \in\{1 \cdots \mathbf{j}\}}$ and $\mathcal{B}_{y}=\left\{y_{j}\right\}_{j \in\{1 \cdots \mathbf{j}\}}$ of $Z$ and $Y$. It is performed by randomly selecting $\mathbf{n}$ samples from $\mathcal{B}_{x}$ to compute samples $z_{j}$ and $y_{j}$ (Efron and Tibshirani, 1998). This should be done without replacement to insure independence of samples of $X$. However, if $\mathbf{i}$ is large, it can also be performed with replacement.

It is assumed that the observed samples $\left\{z_{j}\right\}$ (or $\left\{y_{j}\right\}$ ) are generated from a mixture $\mathcal{P}_{Z}(z \mid \sigma)$ of $\mathcal{P}_{Z}(z \mid \mathcal{C}, \sigma)$ (inliers) and $\mathcal{P}_{Z}(z \mid \overline{\mathcal{C}})$ (outliers). A priori, the proportion of the inliers $\mathcal{P}_{Y}(\mathcal{C})$ in $\mathcal{B}_{y}$ and $\mathcal{P}_{Z}(\mathcal{C})$ in $\mathcal{B}_{z}$ (i.e. when $z_{j}$ and $y_{j}$ are computed using $x_{n} \in$ $\mathcal{C}, \forall n \in[1 ; \mathbf{n}])$ is equal to $\left(\mathcal{P}_{X}(\mathcal{C})\right)^{\mathbf{n}}$. However this proportion can be increased using properties of the data. More precisely, audio and video data present strong spatio-temporal correlations that allow us to assume that data from a neighbourhood belong to the same class $(\mathcal{C}$ or $\overline{\mathcal{C}}$ ) (Dahyot et al., 2004b). Using this assumption, samples for $Y$ and $Z$ are carefully generated in order to limit the proportion of outliers by mis-coupling samples of $X$. As a consequence, it is possible to compute samples of $Y$ and $Z$ such that the proportion of inliers in $\mathcal{B}_{x}, \mathcal{P}_{X}(\mathcal{C})$, is rather close to the proportions of inliers in $\mathcal{B}_{y}$ and $\mathcal{B}_{z}: \mathcal{P}_{X}(\mathcal{C}) \sim \mathcal{P}_{Y}(\mathcal{C}) \sim \mathcal{P}_{Z}(\mathcal{C})$ 


\subsection{Non-parametric estimation}

\subsubsection{Estimating distributions}

From the collection $\mathcal{B}_{y}$ of samples of $Y$, a kernel estimate of the density function $\mathcal{P}_{Y}(y)$ can be computed:

$$
\hat{\mathcal{P}}_{Y}(y)=\frac{1}{\mathbf{i}} \sum_{i=1}^{\mathbf{i}} \frac{1}{h_{y_{i}}} \cdot k\left(\left|\frac{y-y_{i}}{h_{y_{i}}}\right|\right)
$$

with $k(\cdot)$ chosen as a Gaussian $\mathcal{N}(0,1)$. The variable bandwidths $\left\{h_{y_{i}}\right\}_{i=1 \cdots \mathbf{i}}$ are selected automatically following Comanicciu et al. scheme (Comaniciu et al., 2001). The only change concerns the initial setting of the center point bandwidth: instead of the rule of thumb plug-in, the more efficient Sheather-Jones plug-in bandwidth $h_{S J}$ is computed (Sheather, 2004).

\subsubsection{Relation between bandwidths}

For $\alpha=\frac{1}{2}$ (Gaussian distribution for the inliers), using the relation $z_{i}=y_{i}^{2}$ between samples in $\mathcal{B}_{y}$ and $\mathcal{B}_{z}$, variable bandwidths are automatically computed for samples in $\mathcal{B}_{y}$, and then inferred for samples in $\mathcal{B}_{z}$ by:

$$
h_{z_{i}}=h_{y_{i}} \cdot \sqrt{4 y_{i}^{2}+2 h_{y_{i}}}
$$

This relation between $h_{z_{i}}$ and $h_{y_{i}}$ is derived by assuming a Gaussian variable $y \sim \mathcal{N}\left(y_{i}, h_{y_{i}}^{2}\right)$ (from the kernel Gaussian assumption) and then by inferring the variance $h_{z_{i}}^{2}$ of the variable $z=y^{2}$. For other values of $\alpha$, using the relation $z_{i}=y_{i}^{\frac{1}{\alpha}}$, the relation between the bandwidths is approximated using first order Taylor series:

$$
h_{z_{i}}=\frac{1}{\alpha} \cdot y_{i}^{\frac{1}{\alpha}-1} \cdot h_{y_{i}}
$$

\subsubsection{MeanShift}

The closest mode to zero can be computed using mean shift from the minimum value of the samples (Dahyot et al., 2004b; Comaniciu et al., 2001):

$$
\begin{aligned}
& \text { Init } y^{(0)}=\min _{y_{i}} \mathcal{B}_{y} \\
& y^{(m+1)}=\frac{\sum_{i=1}^{\mathrm{i}} \frac{y_{i}}{h_{y_{i}}^{3}} g\left(\left|\frac{y^{(m)}-y_{i}}{h_{y_{i}}}\right|^{2}\right)}{\sum_{i=1}^{\mathrm{i}} \frac{1}{h_{y_{i}}^{3}} g\left(\frac{y^{(m)}-y_{i}}{h_{y_{i}}}\right)} \\
& \text { till convergence } Y_{\max \mathcal{C}}=y^{(\mathbf{m})}
\end{aligned}
$$

with $g(t)=\frac{-k^{\prime}(\sqrt{t})}{2 \sqrt{t}}$. The same procedure is used to estimate $Z_{\max } \mathcal{C}$. Using equation (3.6), estimates for the scale parameter can be inferred. 


\section{Robust regression}

For simplicity, we consider linear regression where observations $\mathcal{B}_{u v}=\left\{\left(v_{i}, u_{i}\right)\right\}_{i=1 \cdots \mathbf{i}}$ follow the linear mapping $v_{i}=u_{i}^{T} \theta+\epsilon_{i}, \forall i$. The joint estimation of $\theta$ and $\sigma$ is performed by using the scale estimate introduced in the previous paragraphs, iteratively with a least squares ${ }^{1}$ estimation of $\theta$ performed on a subset $\mathcal{S}_{u v}$ of $p$ observations ( $p$ is chosen superior or equal to the dimension of $\theta$ ). This is similar to the RANSAC approach with an added scale estimate. The algorithm can be described as:

- Repeat $B$ times (see (Fischler and Bolles, 1981) for the choice of $B$ )

1. Select a subset $\mathcal{S}_{u v}^{(b)}$ of $p$ points randomly selected from $\mathcal{B}_{u v}$,

2. Least Squares estimation of the location parameter $\theta^{(b)}$ on $\mathcal{S}_{u v}^{(b)}$.

3. Compute the residuals $\left\{\epsilon_{i}=v_{i}-u_{i}^{T} \theta^{(b)}\right\}_{i=1 \cdots \mathbf{i}}$, and samples of $Y$ and $Z$.

4. Compute the bandwidths $\left\{h_{y_{i}}\right\}$ and $\left\{h_{z_{i}}\right\}$ as explained in Section 4.2.

5. Estimate the scale parameter $\sigma^{(b)}$ using procedure (4.3) and relations (3.6).

6. Compute the objective function $\mathcal{J}^{(b)}\left(\theta^{(b)}, \sigma^{(b)}\right)$.

- Infer $(\hat{\theta}, \hat{\sigma})$ from $\arg \max \mathcal{J}^{(b)}$ (ou arg min depending on the chosen objective function).

In this article, a similar objective function as Wang et. al has been chosen (Wang and Suter, 2004).

$$
\mathcal{J}(\theta, \sigma)=\frac{\sum_{i=1}^{\mathbf{i}} \mathbf{1}\left\{\left|\epsilon_{i}\right|<2.5 \sigma\right\}}{\mathbf{i} \cdot \sigma}
$$

Note that, with our algorithm, a fixed $\theta$ leads to an estimate of $\sigma$, hence the space of all possibilities $(\theta, \sigma)$ is not fully explored but only the space $\left(\theta, \sigma_{\theta}\right)$. As a consequence the objective function $\mathcal{J}$ has only $\theta$ as a variable. Some representations of the objective function are presented in the experimental results in Section 6.2. Similarily to the Hough transform (Goldenshluger and Zeevi, 2004), the problem of recovering several models (or multi-lines when $\theta$ is of dimension $p=2$ ) is to find all maxima (or minima) in $\mathcal{J}$.

\section{Experimental results}

Assessment of the robustness of the proposed method is done using simulations. Those simulations are performed under certain conditions explained below. That reflect real situations of interest encountered in data analysis. In Section 6.1, two scenarios are tried : outliers with uniform distribution and pseudo-outliers with a Gaussian distribution. Results for robust regression are reported in Section 6.2.

\footnotetext{
${ }^{1}$ The inliers are assumed here to be Gaussian: $\alpha=0.5$.
} 


\subsection{Scale estimate}

In the following experiments, we chose $\alpha=\frac{1}{2}$, or a Gaussian distribution for the inliers, $\mathbf{i}=1000$ (cardinal of $\mathcal{B}_{Y}$ and $\mathcal{B}_{Z}$ ), the groundtruth scale parameter $\sigma=2$ and the degree of freedom $\mathbf{n}=3$ :

- $\forall n=1, \cdots, \mathbf{n}$, sets $\mathcal{B}_{x_{n}}$ are used to compute sets $\mathcal{B}_{y}$ and $\mathcal{B}_{z}$ such that the proportion of inliers $\forall n, \mathcal{P}_{X_{n}}(\mathcal{C})=\mathcal{P}_{Y}(\mathcal{C})=\mathcal{P}_{Z}(\mathcal{C})$. Outliers follow a uniform distribution $\forall n, \mathcal{P}_{X_{n}}(x \mid \overline{\mathcal{C}})=\mathcal{U}([-50 ; 50])$.

- Pseudo-outliers follows a Gaussian distribution with the same variance as the inliers and a mean $\mu$. The proportion of the inliers is fixed such that $\forall n, \mathcal{P}_{X_{n}}(\mathcal{C})=$ $\mathcal{P}_{Y}(\mathcal{C})=\mathcal{P}_{Z}(\mathcal{C})=0.1$.

\subsubsection{Robustness to outliers}

Figure 2 presents the mean of the estimates depending on the proportion of the inliers computed over 50 tries. As the proportion of inliers increases, the accuracy improves. Althought $\sigma_{Z}$ is less accurate than $\sigma_{Y}$, it shows a better robustness to the proportion of outliers. It is understood that the peak localisation for the estimation is easier performed on the distribution of $Z$ than $Y$. In fact, when too many outliers occur, the inlier peak is not anymore distinguishable in the pdf of $Y$.
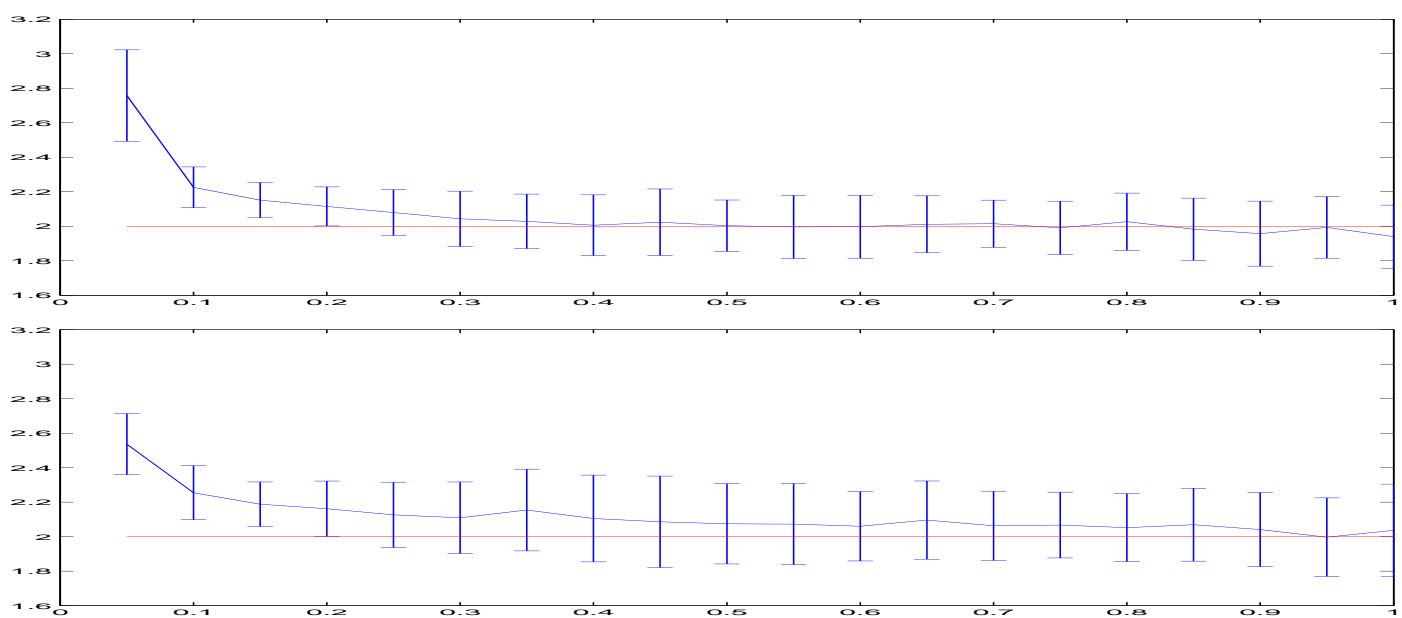

Figure 2: Robustness to outliers: Estimates $\sigma_{Y}$ (top) and $\sigma_{Z}$ (bottom) with standard deviation, w.r.t. the proportion of inliers $\mathcal{P}_{Y}(\mathcal{C})=\mathcal{P}_{Z}(\mathcal{C})$. The red line corresponds to the groundtruth scale parameter.

\subsubsection{Robustness to pseudo-outliers}

Figure 3 presents the mean of the scale estimates $\sigma_{Y}$ and $\sigma_{Z}$ with standard deviation, depending on the mean of the pseudo-outliers, computed over 50 tries. The estimate $\sigma_{Y}$ is shown to be accurate when the mean $\mu$ of the pseudo-outliers is above $3 \sigma$. The estimate $\sigma_{Z}$ is rather accurate already when $\mu>2.5 \sigma$. Figure 4 presents the distributions 

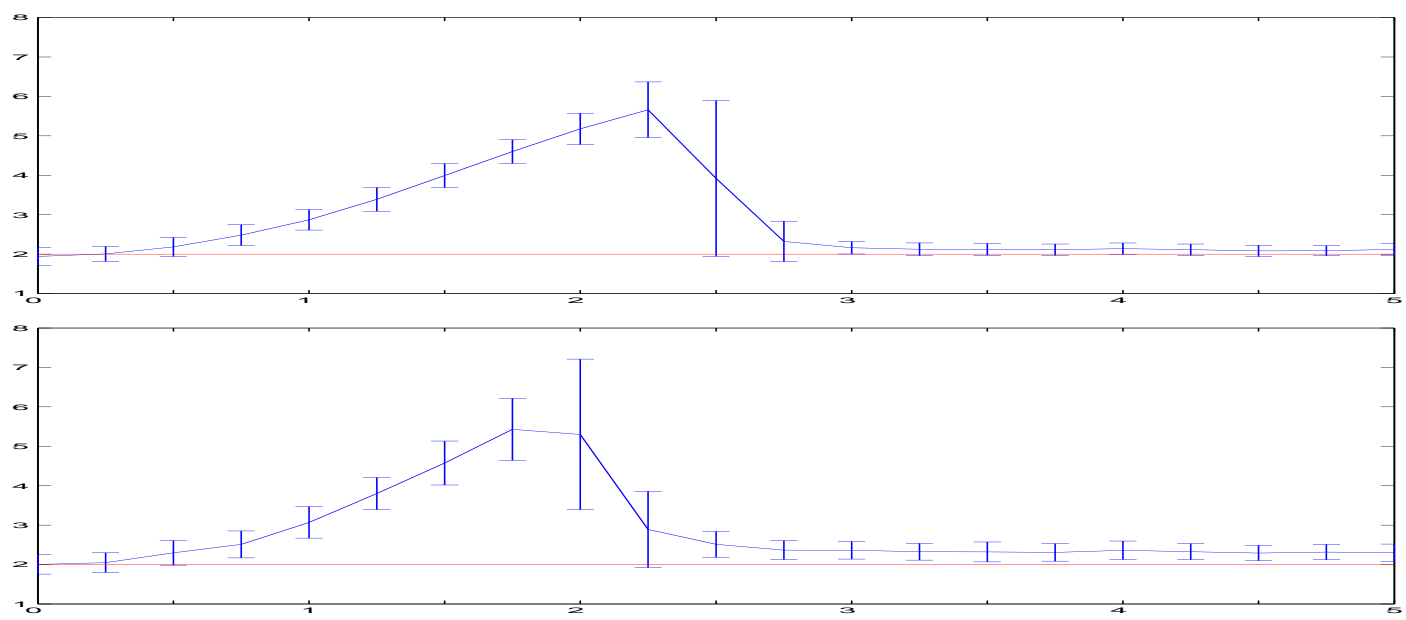

Figure 3: Robustness to pseudo-outliers: Estimates $\sigma_{Y}$ (top) and $\sigma_{Z}$ (bottom) with standard deviation, w.r.t. the mean of the Gaussian of pseudo-outliers expressed as a multiplicative of $\sigma$ (i.e. abscissa equal to 2 means $\mu=2 \sigma$ ).

$\mathcal{P}_{X}(x \mid \sigma), \mathcal{P}_{Y}(y \mid \sigma)$ and $\mathcal{P}_{Z}(z \mid \sigma)$ for different values of the mean $\mu$ of the pseudo-outliers. The distributions are estimated using the kernel modelling as explained in Section 4.2. When the inlier and pseudo-outlier Gaussians are too close, their respective maxima are not anymore distinguishable.

\subsubsection{Remarks}

Similar results have been obtained for various inliers distribution i.e. different values of $\alpha$ (in between 0.5 to 1 ) and $\mathbf{n}=2,3$. In practice, the choice of $\mathbf{n}$ should be as low as possible to simplify the computation of samples of $Y$ and $Z$.

\subsection{Robust regression}

In a similar experience as in (Wang and Suter, 2004), line parameters are estimated iteratively with the scale parameter of the residuals (Miller and Stewart, 1996; Wang and Suter, 2004), following the procedure described in Section 5. Figure 5 shows the result of the estimation when $90 \%$ of outliers, uniformly distributed, appear in the observations. This result has been obtained using the estimate $\sigma_{Y}$ and $\sigma_{Z}$. Several simulations have been run on different randomly generated sets of observations. Ten estimates of the line are reported on the graph.

The previous experience is repeated with an added line of 50 points generated with the equation $u=v$. Figure 6 shows the observations where alignements are barely distinguishable (left). Both lines can however be recovered in analysing maxima of the objective function. Figure 7 presents the objective function $\mathcal{J}$ as a function of the 2dimensional $\theta$. Two peaks are clearly localised at the neighborhouds of $\theta=(1,0)$ and $\theta=(-1,100)$, corresponding to the two lines coefficients. Numerically the first two maxima computed correspond to $\hat{\theta}=(-0.9710,98.4041)$ and $\hat{\theta}=(1.0135,-0.1504)$. 

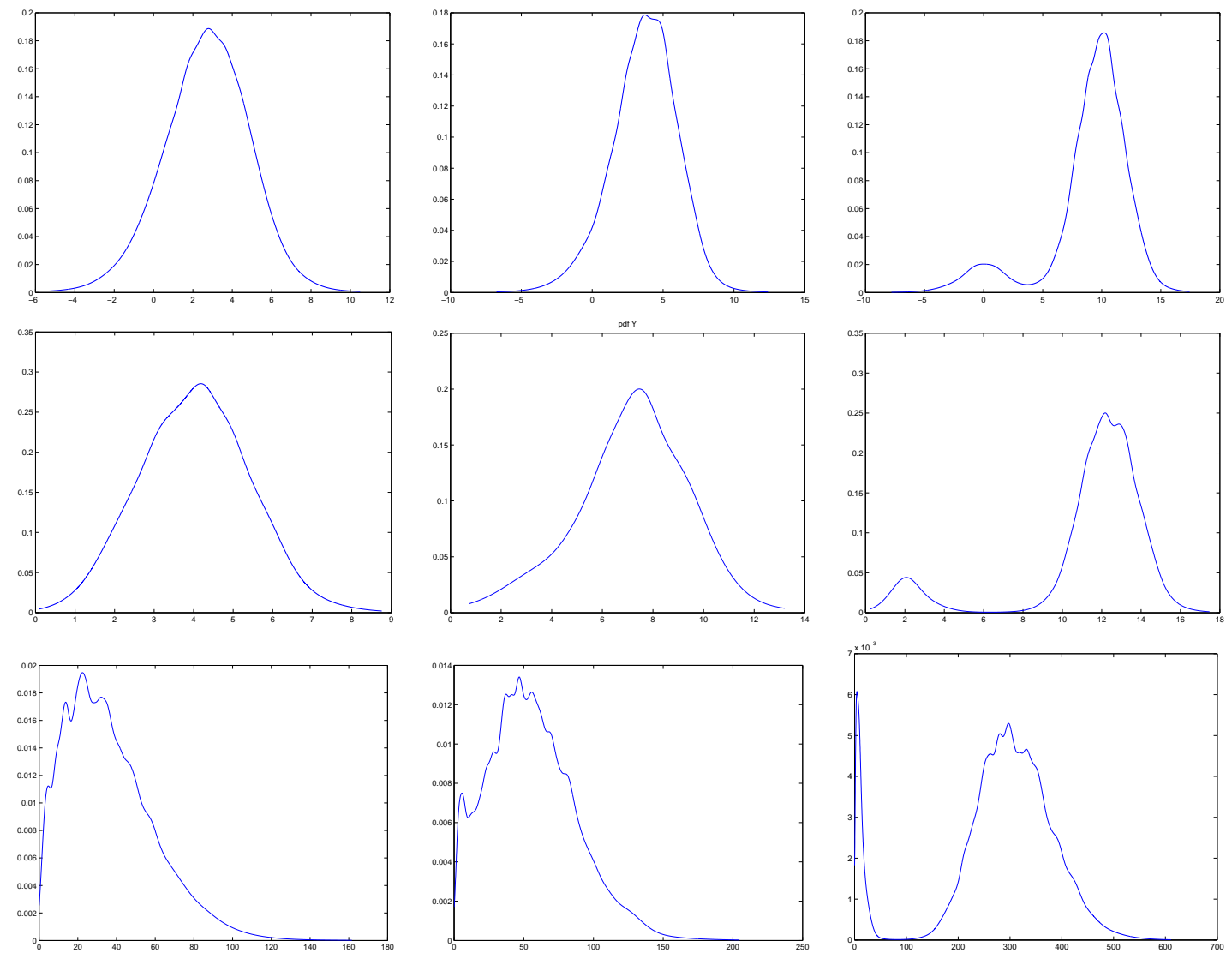

$$
\mu=1.5 \sigma
$$

$\mu=2 \sigma$

$$
\mu=5 \sigma
$$

Figure 4: Distributions $\mathcal{P}_{X}(x \mid \sigma)$ (top), $\mathcal{P}_{Y}(y \mid \sigma)$ (middle), $\mathcal{P}_{Z}(z \mid \sigma)$ (bottom). The relevant maximum for the estimation of the scale parameter becomes distinguishable after $\mu>3 \sigma$.


Figure 5: Robust line fitting. Inliers: 50 points on a line $x \in[0-100], u=-v+100$ and $\sigma=1$; Outliers: 450 points uniformaly distributed (Wang and Suter, 2004). The red line represents the groundtruth, and the green lines represent the estimates perform on 10 trials using $\sigma_{Y}$ (left) and $\sigma_{Z}$ (right). 

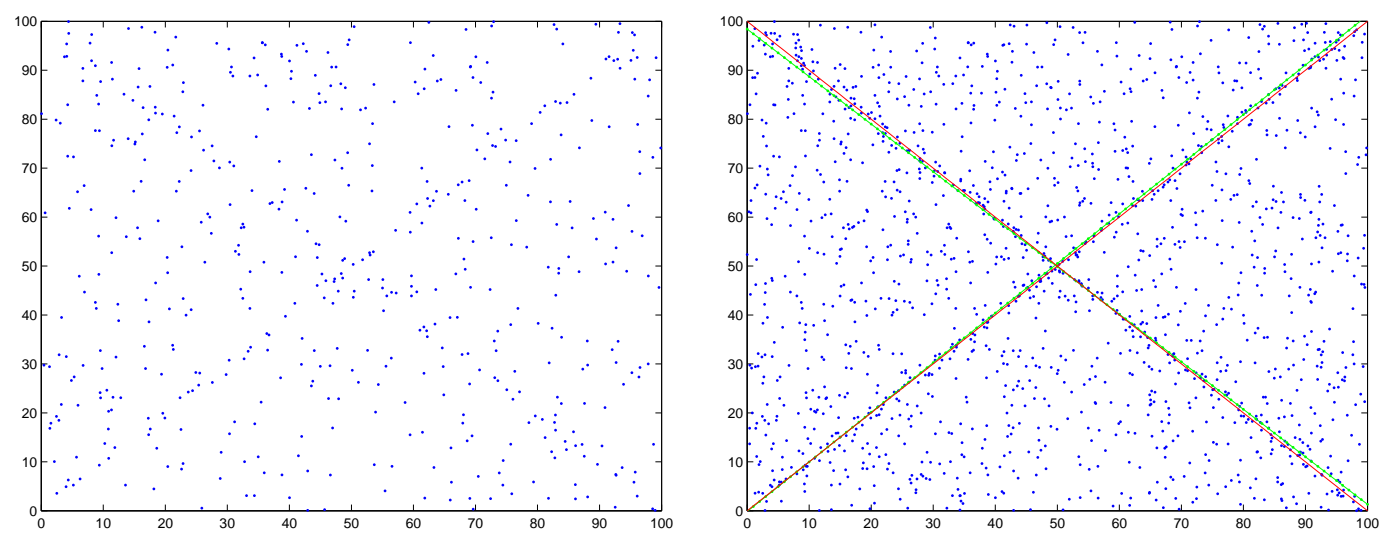

Figure 6: A two line fitting problem: Observations and estimated lines (green lines) with ground truth (red).

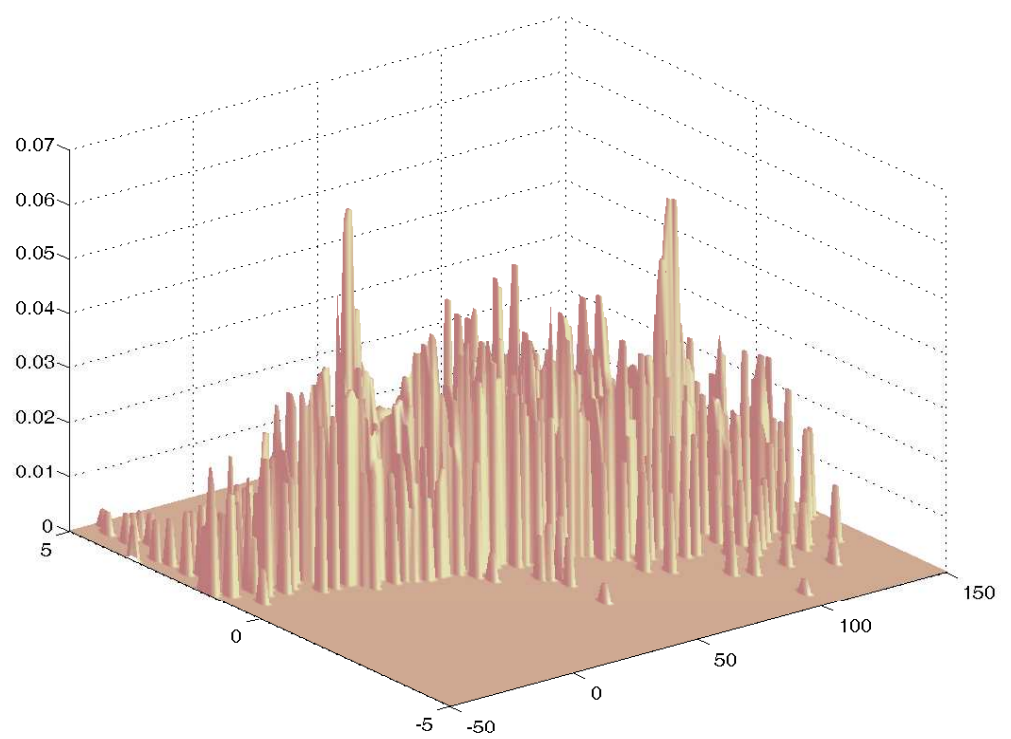

Figure 7: Objective function computed during the estimation. Two lines are present in the observations with parameter $\theta=(-1,100)$ and $\theta=(1,0)$. Both can be recovered by localising the two main peaks.

Figure 7 presents the computed objective function as it is performed in the robust estimation proposed in paragraph 5. For comparison, we computed the objective function for all values $(\theta, \sigma)$ on a finite domain and the graph is reported in figure 8 .

\subsection{Applications in computer vision}

Application to edge detection in images of this scale parameter estimation is suggested (Rosin, 1997). Applications to silence detection in audio data streams, and region segmentation in colour images have been proposed in (Dahyot et al., 2004b). We consider here the problem of robust object recognition (Leonardis and Bischof, 2000) in colour images (Dahyot et al., 2000). 


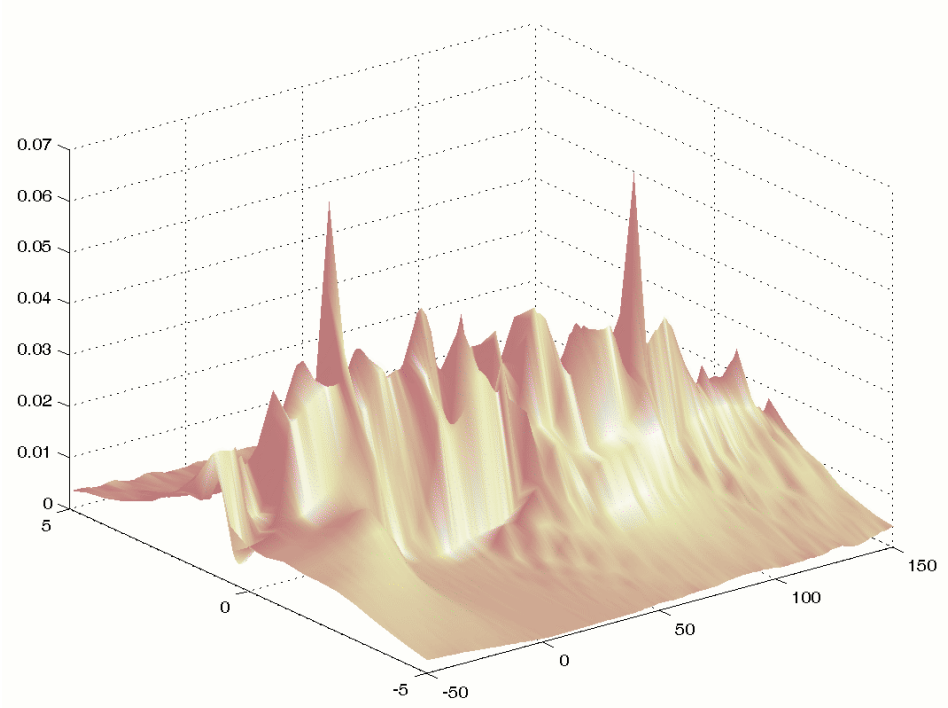

Figure 8: Simulated objective function.

A linear mapping is estimated by applying Principal Component Analysis (Dahyot et al., 2000) on 72 training colour images (image pixel values are arranged in a vector in lexicographic order) representative of an object class (cf. fig. 9). Eigenvectors associated with the highest eigenvalues summarize the informative visual content over the training set. The representative eigenspace is chosen of dimension 3 for this experiment. Figure

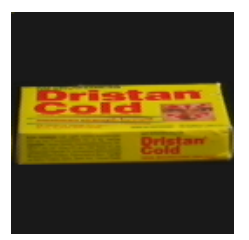

$t_{0}$

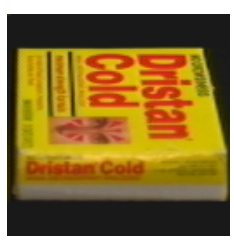

$t_{90}$

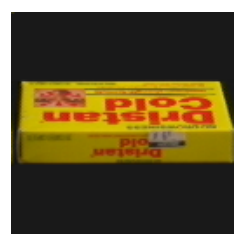

$t_{180}$

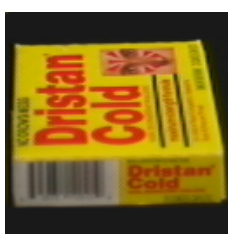

$t_{270}$

Figure 9: Example of training colour images for one object varying under different viewpoints (Nene et al., 1996).

10 presents the eigenbasis including the mean image and the first three eigenvectors. The last image is the reconstruction of the template $t_{0}$ performed on this basis. Considering an

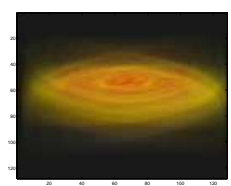

$\mu$

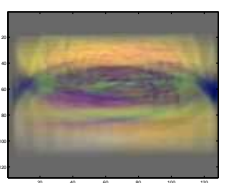

$\mathbf{u}_{1}$

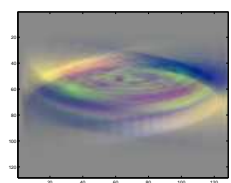

$\mathbf{u}_{2}$

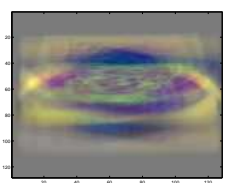

$\mathbf{u}_{3}$

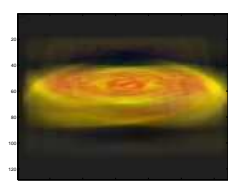

$\hat{t}_{0}$

Figure 10: Mean and eigenvectors selected to represent the object class, and the reconstruction of one of the training template in this eigenspace. 
unknown observation (image), its recognition consists in two tasks. The first is to estimate its coordinate on the eigenspace (corresponding to the 3-dimensional location parameter $\theta$ ). Then the recognition is completed by comparing this estimate with the coordinates indexing the training images. The estimation problem can be written as follows:

$$
\left\{\begin{array}{c}
R_{i}=\mu_{i}^{R}+\left(\mathbf{u}_{i}^{R}\right)^{T} \theta+\epsilon_{i}^{R} \\
G_{i}=\mu_{i}^{G}+\left(\mathbf{u}_{i}^{G}\right)^{T} \theta+\epsilon_{i}^{G} \\
B_{i}=\mu_{i}^{B}+\left(\mathbf{u}_{i}^{B}\right)^{T} \theta+\epsilon_{i}^{B}
\end{array}\right.
$$

where $\left(R_{i}, G_{i}, B_{i}\right)$ are the colour values at the pixel $i$ in the observed image, and $\left(\mu_{i}^{R}, \mu_{i}^{G}, \mu_{i}^{B}\right)$ and $\left(\mathbf{u}_{i}^{R}, \mathbf{u}_{i}^{G}, \mathbf{u}_{i}^{B}\right)$ are the mean and the eigenvector values at pixel $i$ (from the learning). Residuals on each colour band are independent $(\mathbf{n}=3)$. Noise is assumed Gaussian ( $\alpha=0.5$ ) and outliers typically occur because of a changing background or partial occlusions.

The estimation of $\theta$ is performed as described in Section 5. A comparison with robust recognition using M-estimators (Dahyot et al., 2000) is proposed and several results are presented in figure 11. Observations present the object with added Gaussian noise, different colour background and possible partial occlusions. The observations with a yellow background (same yellow as the main colour of the object itself) is tricky for the M-estimator based recognition method. In fact, M-estimation tries to match as many pixels as possible in the observation and consequently matches templates with the highest number of yellow pixels. Our method however, with its objective function taking into account both the scale and location parameter estimates from the current observation, gives a more accurate match for recognition.

\section{Conclusion}

The main idea of this article is to consider the generation of new variables whose distributions show relevant properties for the estimation of an unknown parameter of the original variable. In particular, modes or maxima related to the unknown parameter can be located using non-parametric modelling. Accuracy of this estimation relies on the accuracy of the estimated density function, here performed by nonparametic modelling using kernels. The association with location parameter estimation performs very well in terms of robustness to outliers. The proposed method is fully automatic, one drawback being the computation of samples of $Y$ or $Z$ that must be carefully done to limit the proportion of outliers.

\section{Acknowledgments}

This work has been funded by the European Network Of Excellence on Multimedia Understanding through Semantics, Computation and Learning, MUSCLE FP6-5077-52 (www.muscle-noe.org). 


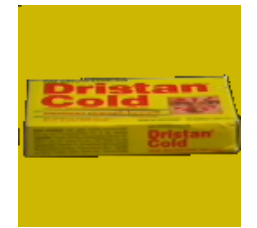

$\mathcal{O}_{1}$

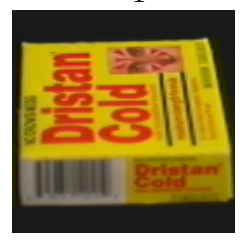

$t_{270}$

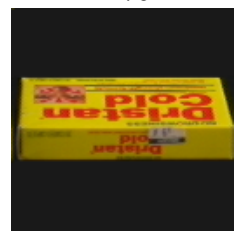

$t_{180}$

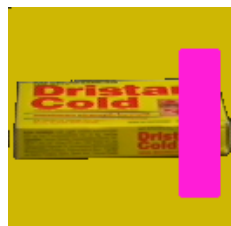

$\mathcal{O}_{2}$

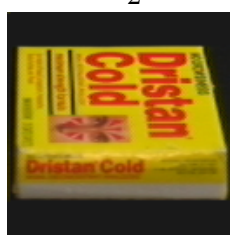

$t_{90}$

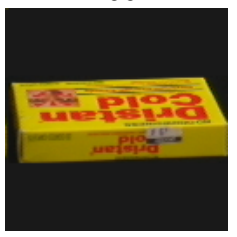

$t_{175}$

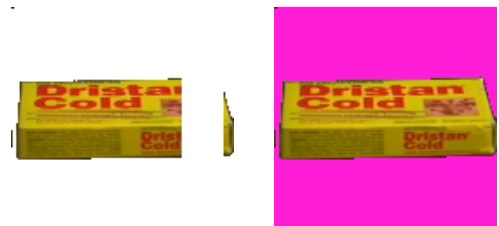

$\mathcal{O}_{4}$
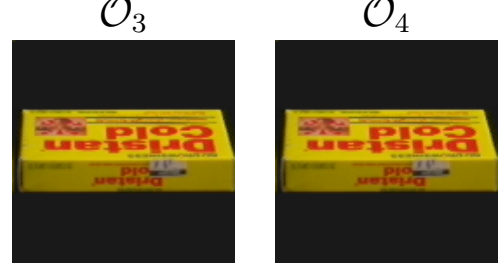

$t_{180}$

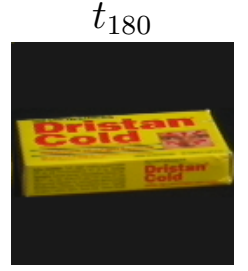

$t_{355}$

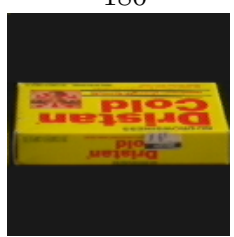

$t_{180}$

Figure 11: Observations (top), recognition performed with M-estimators as in (Dahyot et al., 2000) (middle), recognition with simultaneous robust estimation of the scale and location parameters (bottom).

\section{References}

[1] Aiazzi, B., Alparone, L., and Baronti, S. (1999): Estimation based on entropy matching for generalized gaussian pdf modeling. IEEE Signal Processing Letters, 6.

[2] Black, M. J. and Jepson, A. D. (1998): Eigentracking: Robust matching and tracking of articulated objects using a view-based representation. International Journal on Computer Vision, 26, 63-84.

[3] Bouthémy, P., Gelgon, M., and Ganansia, F. (1999): A unified approach to shot change detection and camera motion characterization. IEEE Transactions on Circuits and Systems for Video Technology, 9, 1030-1044.

[4] Chen, H. and Meer, P. (2003): Robust regression with projection based mestimators. In International Conference on Computer Vision, 878-885, Nice, France.

[5] Comaniciu, D., Ramesh, V., and Meer, P. (2001): The variable bandwidth mean shift and data-driven scale selection. In International Conference on Computer Vision, 438-445, Vancouver, Canada.

[6] Dahyot, R., Charbonnier, P., and Heitz, F. (2000): Robust visual recognition of colour images. In IEEE proceedings of the conference on Computer Vision and Pattern Recognition, volume 1, 685-690, Hilton Head Island, USA. 
[7] Dahyot, R., Charbonnier, P., and Heitz, F. (2004a): A bayesian approach to object detection using probabilistic appearance-based models. Pattern Analysis and Applications, 7, 317-332.

[8] Dahyot, R., Rea, N., Kokaram, A., and Kingsbury, N. (2004b): Inlier modeling for multimedia data analysis. In IEEE International Workshop on MultiMedia Signal Processing, Siena Italy.

[9] Efron, B. and Tibshirani, R. J. (1998): An Introduction to the Bootstrap. Chapman \& Hall/CRC.

[10] Fischler, M. A. and Bolles, R. C. (1981): Random sample consensus: a paradigm for model fitting with applications to image analysis and automated cartography. Commun. ACM, 24, 381-395.

[11] Goldenshluger, A. and Zeevi, A. (2004): The hough transform estimator. The Annals of Statistics, 32.

[12] Hampel, F. R., Ronchetti, E. M., Rousseeuw, P. J., and Stahel, W. A. (1986): Robust Statistics : The Approach Based on Influence Functions. John Wiley and Sons.

[13] Hasler, D., Sbaiz, L., Süsstrunk, S., and Vetterli, M. (2003): Outlier modeling in image matching. IEEE Transactions on Pattern Analysis Machine Intelligence, 25, 301-315.

[14] Huber, P. (1981): Robust Statistics. John Wiley and Sons.

[15] la Torre, F. D. and Black, M. J. (2001): Robust principal component analysis for computer vision. In Proceedings of the International Conference on Computer Vision, Vancouver, Canada.

[16] Leonardis, A. and Bischof, H. (2000): Robust recognition using eigenimages. Computer Vision and Image Understanding, 78, 99-118.

[17] Marazzi A., R. C. (1996): Implementing m-estimators of the gamma distribution. Robust Statistics, Data Analysis, and Computer Intensive Methods, in Honor of Peter Huber's 60th Birthday, 277-297.

[18] Miller, J. V. and Stewart, C. V. (1996): Muse: Robust surface fitting using unbiased scale estimates. In IEEE Conference on Computer Vision and Pattern Recognition, 300-306.

[19] Nene, S. A., Nayar, S. K., and Murase, H. (1996): Columbia object image library (coil-100). Technical Report CUCS-006-96, Department of Computer Science, Columbia University.

[20] Odobez, J.-M. and Bouthémy, P. (1995): Robust multiresolution estimation of parametric motion models. Journal of Visual Communication and Image Representation, 6, 348-365. 
[21] Rosin, P. L. (1997): Edges: saliency measures and automatic thresholding. Machine Vision and Applications, 9, 139-159.

[22] Sheather, S. J. (2004): Density estimation. Statistical Science, 19, 588-597.

[23] Stewart, C. (1999): Robust parameter estimation in computer vision. SIAM Reviews, 41, 513-537.

[24] Wang, H. (2004): Robust Statistics for Computer Vision: Model fitting, Image Segmentation and Visual Motion Analyis. PhD thesis, Monash University, Clayton Victoria, Australia.

[25] Wang, H. and Suter, D. (2004): Robust adaptive-scale parametric model estimation for computer vision. Transactions on Pattern Analysis and Machine Intelligence, 26. 